Note

\title{
The interaction of methylmercury with lysosomes from rat liver
}

\author{
Marcantonio Bragadin $^{\mathrm{a}, *}$, Guido Scutari ${ }^{\mathrm{b}}$, Sabrina Manente ${ }^{\mathrm{a}}$, Antonio Toninello ${ }^{\mathrm{b}}$ \\ a Dipartimento di Scienze Ambientali, Università di Venezia, DD 2137, 30123 Venezia, Italy \\ b Dipartimento di Scienze Biomediche Sperimentali, Università di Padova, Viale G. Colombo 3, 35121 Padova, Italy
}

Received 8 January 2002; accepted 20 February 2002

\begin{abstract}
The interactions of methylmercury with rat liver lysosomes were investigated. The results indicate that methylmercury enhances the proton permeability in membranes. Since this uncoupling mechanism necessarily implies the transport of a charged ion (in this case $\mathrm{CH}_{3} \mathrm{Hg}^{+}$) through the membrane, this fact can help to explain why a preferential target of methylmercury are nerve cells, whose plasma membrane potential has the highest value, when compared with other cells. C) 2002 Elsevier Science B.V. All rights reserved.
\end{abstract}

Keywords: Lysosomes; Methyl complexes; Mercury complexes

\section{Introduction}

The presence of methylmercury in natural waters is a problem of great environmental concern since this compound possesses a high toxicity very well documented in animals and humans [1-6].

Methylmercury is prevalently neurotoxic and studies have been performed in cells and in subcellular structures [7-10] in order to establish the molecular mechanism (or mechanisms) which is responsible for the toxicity in whole animals.

Methylmercury is reported to increase cytosolic $\mathrm{Ca}^{++}$concentration in rat cerebrum synaptosomes [7], in PC12 cells [8], and in rat T lymphocytes [9]. In other cellular systems, the inhibition of ATP synthesis and apoptosis have also been reported [10,11].

Among the subcellular structures, mitochondria have been extensively investigated since a damage on mitochondria, which produce ATP for the cell, gives rise to a corresponding cell damage [12-17]. In mitochondria, methylmercury interacts with all the mechanisms, which are involved in ATP synthesis. In fact, it has been reported that methylmercury inhibits the respiratory chain $[14,15]$, and, in the same way as uncoupler, it

\footnotetext{
* Corresponding author. Tel.: +39-041-257 8507; fax: +39-041-257 8584

E-mail address: bragadin@unive.it (M. Bragadin).
}

collapses $\Delta \psi$ and causes a $\mathrm{Ca}^{++}$release [14-16]. Furthermore, it induces a cytochrome $c$ release [17] and the opening of a transition pore [18]. In mitochondria, therefore, it is difficult to establish the effective toxic effect (the lowest dose which causes a failure in ATP synthesis) since many interaction mechanisms are present. Furthermore, the effective toxic effect in cells and, in particular, the reason why nerve cells are the preferential target for methylmercury has not yet been established.

In this paper our attention is focused on lysosomes. Up to now, the interactions of methylmercury with these organelles have never been examined and our aim is to verify whether a particular transport system of this compound is present in the lysosomal membrane.

Moreover, a study of a membrane transport mechanism in lysosomes offers some advantages since, in this biological system, many functions such as the respiratory chain, selective transition pore and antiporters are absent or present in a limited number. This facilitates the approach and could permit to propose a general peculiar behaviour also for other membranes. The results, in reality, indicate that methylmercury behaves as an uncoupler. This behaviour, being a membrane property, could explain not only one aspect of the toxicity of methylmercury since uncouplers are toxic compounds, but in particular the prevalent neurotoxicity of the compound. 


\section{Materials and methods}

Rat liver lysosomes were prepared according to Sawant et al. [19] and resuspended before use at $0{ }^{\circ} \mathrm{C}$ in a medium (mother solution) containing $0.25 \mathrm{M}$ sucrose, $10 \mathrm{mM}$ Mops-Hepes $\mathrm{pH} 7.4$ and $5 \mathrm{mM}$ $\mathrm{MgSO}_{4}$. Acridine Orange (AO) (Merck) was purified according to Pal and Schubert [20]. Aliquots of lysosomes were withdrawn from the mother solution and added to the operating medium. The uptake of AO by lysosomes was monitored spectrophotometrically at room temperature using a Jenway 6400 spectrophotometer, by measuring the change in dye absorbance at $492 \mathrm{~nm}$. After the addition of the lysosomes and AO to the operating medium (final concentration of lysosomes $0.2 \mathrm{mg} \mathrm{ml}^{-1}$ ), the spectrophotometer was adjusted to 0 absorbance. The protein concentration was determined by the Lowry method [21].

ATPase inhibition measurements were performed following the procedure suggested by Nishimura and Cahnce [22] by means of a pH meter (PHM 84 Radiometer Copenaghen). The medium contained Oligomycin in order to prevent spurious mitochondrial contaminations and $100 \%$ inhibition of the rate of ATP hydrolysis was measured in the presence of bafilomycin.

Determination of the concentration of $\mathrm{CH}_{3} \mathrm{HgCl}$ in the supernatant of energized lysosomes. The suspension containing energized lysosomes (medium and conditions as in Fig. 1) was centrifuged at $14.000 \mathrm{rpm}$ by means of a Sorvall centrifuge RC2B, rotor SS34. The mercury content in the supernatant was obtained by flow injection (Perkin-Elmer 3.100) cold vapor atomic absorbtion Spectroscopy (Perkin-Elmer FIAS 100) using $\mathrm{SnCl}_{2}$ as reducent.

All the reagents were of an analytical grade. The bafilomycin, duramycin, valinomycin and 2,4-dinitrophenol (DNP) were purchased by Sigma (Milan).

\section{Results and discussion}

In lysosomes the $\mathrm{pH}$ in the matrix is acid, about 5.2 as a consequence of a Donnan effect [23]. The lysosomes possess an ATP-driven proton pump, which, in the presence of ATP, pumps protons into the matrix. The acidification in lysosmes can easily be monitored using the AO dye [24]. This dye is a weak permeant base and, following the classical mechanism of weak bases, it crosses the membrane as an electroneutral compound and accumulates in the matrix, since the $\mathrm{pH}$ is acid inside. The accumulation gives rise to a chemical shift [25]. Therefore, the accumulation and the internal acidification can easily be monitored by following the absorbance quenching at $492 \mathrm{~nm}$, as shown in Fig. 1.

In Fig. 1, the absorbance quenching signal before the addition of ATP is due to the non-energized entry of
$\mathrm{AO}$, as a consequence of the internal $\mathrm{pH}$ of 5.2 as mentioned above. The addition of ATP causes a further acidification, since the ATP-driven proton pump pumps the protons into the matrix, but the acidification is appreciable and monitorable only if $\mathrm{Cl}^{-}$ions are present in the medium. This is due to the fact that the pumping of protons induces a positive-inside potential, which is a force that opposes further significant acidification. In the presence of $\mathrm{Cl}^{-}$ions, a selective channel allows for the $\mathrm{Cl}^{-}$uptake by the positive-inside potential. The $\mathrm{Cl}^{-}$entry neutralizes, although not completely (see below) the internal potential, and, as a consequence, the protons are 'easily' pumped from the pump into the matrix without the opposing force of the positive-inside potential [26]. Under these conditions the internal $\mathrm{pH}$ is about 4.5 [26].

Analogously, since the mechanism which regulates internal acidification implies the presence of a positiveinside potential which is an opposing force, a marked and monitorable ATP dependent acidification is observed in a medium not containing $\mathrm{Cl}^{-}$, but containing valinomycin, the potassium carrier [27]. Under these experimental conditions, valinomycin allows for a potassium efflux from the matrix (the matrix contains $0.1 \mathrm{M}$ potassium) to the suspending medium, which is equivalent (with regard to the electrical balance) to a $\mathrm{Cl}^{-}$uptake (Fig. 2)

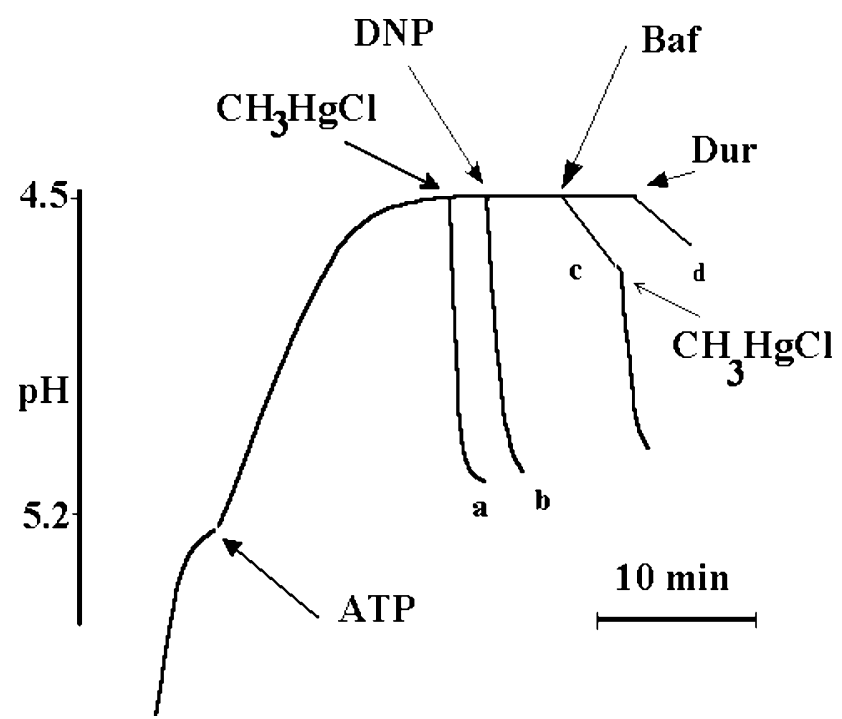

Fig. 1. Alkalization induced by methylmercury or by DNP, bafilomycin (Baf) and duramycin (Dur) in energized lysosomes resuspended in a chloride medium. Medium composition: $125 \mathrm{mM} \mathrm{KCl,} 20 \mathrm{mM}$ Hepes $\mathrm{pH} 7.4,5 \mathrm{mM} \mathrm{MgCl}$. Immediately after addition to the medium (2.5 $\mathrm{ml}$ ) of lysosomes and $\mathrm{AO}$ (final concentration $3 \mathrm{mg} \mathrm{ml}^{-1}$ and $5 \mu \mathrm{M}$, respectively) the instrument was adjusted to zero absorbance. Under these conditions, the trace, before the addition of ATP, measures the internal $\mathrm{pH}$ of non-energized lysosomes ( $\mathrm{pH}$ 5.2). After the addition of ATP $(2 \mathrm{mM})$, a further acidification occurs. In a steady state, the traces show the addition of: $20 \mu \mathrm{M} \mathrm{CH}_{3} \mathrm{HgCl}$ (trace a), $40 \mu \mathrm{M}$ DNP (trace b), $50 \mathrm{nmol}$ bafilomycin (trace c), $550 \mathrm{ng}$ duramycin (trace d). 


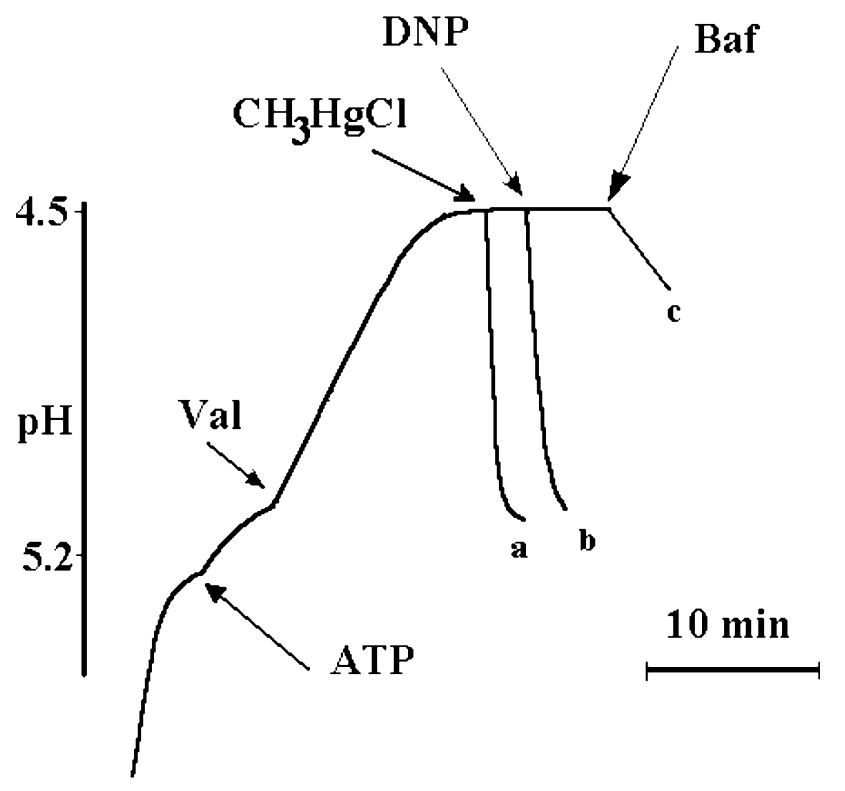

Fig. 2. Alkalization induced by methylmercury or by DNP and bafilomycin (Baf) in energized lysosomes resuspended in a chloridefree medium and in the presence of valinomycin. Medium composition: $25 \mathrm{mM}$ sucrose, $20 \mathrm{mM}$ Hepes $\mathrm{pH} 7.4,5 \mathrm{mM} \mathrm{MgSO}_{4}$. The instrument was adjusted, before the addition of ATP to the medium $(2.5 \mathrm{ml})$ to zero absorbance, with the same concentration of lysosomes and $\mathrm{AO}$ as in Fig. 1. After the addition $(2 \mathrm{mM})$ of ATP, $50 \mathrm{nmol}$ of valinomycin (Val) were added. In a steady state, the traces indicate the addition of: $20 \mu \mathrm{M} \mathrm{CH} \mathrm{CH}_{3} \mathrm{HgCl}$ (trace a) or $40 \mu \mathrm{M}$ DNP (trace b), 50 nmol bafilomycin (Baf) (trace c).

In all cases (in the presence of chloride or valinomycin in the medium) about 10 min after addition of ATP, the system is in a steady state condition, in which the internal $\mathrm{pH}$ is about 4.5 [25] and the internal potential $\Delta \psi$ is low, about $+40 \mathrm{mV}$ [28]. If, under these conditions, methylmercury is added, a rapid alkalinization occurs (Figs. 1 and 2, traces a).

In general, a rapid alkalinization can be due to many factors:

a) An uncoupling effect [29]: The lysosomal membrane is not permeable to protons. If a protonophor (or uncoupler) is added, internal acidification occurs. Figs. 1 and 2 (traces b) show examples of (rapid) internal alkalinization, induced by DNP, which is a classical uncoupler. DNP permits the permeation of protons since it enters the matrix as a phenate negative ion, the driving force being the positiveinside potential. Once inside, it is extruded as an electroneutral undissociated compound, since DNP is a weak acid and the $\mathrm{pH}$ is acid inside. The balance of this cyclic mechanism is the transport of a proton through the membrane during any cycle. This mechanism, being cyclic, well explains the low doses of uncoupler ( $40 \mu \mathrm{M}$ in the case of DNP) required to obtain a rapid and complete internal $\mathrm{pH}$ alkalinization. b) Weak base effect [30]: Weak bases such as ammonia enter the membrane and accumulate inside, since the $\mathrm{pH}$ is acid inside. The alkalinization occurs only in the presence of high doses of a weak (permeant) base, above millimolar [30].

c) Inhibition of the ATPase [31]: Many chemical compounds inhibit the ATPase. Bafilomycin is a potent inhibitor [31]. Its addition in a steady state causes a slow alkalinization (Figs. 1 and 2, traces c).

d) Inhibition of the $\mathrm{Cl}^{-}$channel [32]: Duramycin is a potent inhibitor of the $\mathrm{Cl}^{-}$channel [32]. Its addition in a steady state causes a slow alkalinization (Fig. 1, trace d).

e) Presence of an antiporter system, such as an $\mathrm{OH}^{-}$/ $\mathrm{Cl}^{-}$exchanger [33,34]. This situation has been proposed as an explanation for the toxicity of many organometal compounds, such as alkyl ${ }_{3} \mathrm{SnCl}$ and alkyl $_{3} \mathrm{PbCl}[33,34]$. This mechanism is, in theory, proposable for methylmercury, since it forms the $\mathrm{CH}_{3} \mathrm{HgCl}$ complex $[35,36]$.

In solution, methylmercury chloride gives rise to the following equlibria: [35,36]

$$
\begin{aligned}
& \mathrm{CH}_{3} \mathrm{HgCl} \rightleftarrows \mathrm{CH}_{3} \mathrm{Hg}^{+}+\mathrm{Cl}^{-} \quad(\mathrm{m}) \\
& \mathrm{CH}_{3} \mathrm{Hg}^{+}+\mathrm{H}_{2} \mathrm{O} \rightleftarrows \mathrm{CH}_{3} \mathrm{HgOH}+\mathrm{H}^{+}
\end{aligned}
$$

The dose of methylmercury necessary to obtain a fast alkalinization such as that indicated in Figs. 1 and 2 is in the order of $1 \mu \mathrm{M}$. Therefore, a weak-base mechanism (mechanism b), as methylmercury is a weak base (equilibrium n) should be excluded, even if theoretically possible, since weak bases require high doses, of above $10 \mathrm{mM}$.

Analogously, even though we cannot exclude the fact that methylmercury inhibits ATPase (see below), such behaviour should be excluded, since the ATPase inhibition induced by bafilomycin [31], which is a potent and instantaneous ATPase inhibitor, gives rise to a slow alkalinization, even slower than that induced by methylmercury.

In the same way, even though methylmercury inhibits the $\mathrm{Cl}^{-}$channel, this is not the reason why alkalinization is induced, since Fig. 1 shows that the addition of duramycin, the chloride channel inhibitor [32] gives rise to a slow alkalinization.

In any case, methylmercury also induces alkalinization in a chloride free medium, as shown in Fig. 2. Therefore, even though we cannot exclude an inhibitory effect on the chloride channel, this is not the cause of the rapid alkalinization shown in Figs. 1 and 2.

An antiporter mechanism (mechanism e) has been proposed as an explanation of the toxicity mechanism of $\mathrm{alkyl}_{3} \mathrm{SnCl}$ and $\mathrm{alkyl}_{3} \mathrm{PbCl}$ in mitochondria $[32,33]$. Since methylmercury forms stable chloride complexes (eq $\mathrm{m}$ ), this mechanism can explain the behaviour in Fig. 
1, but not in Fig. 2 since in Fig. 2, the chloride is absent from the medium.

On the basis of these observations, we have concluded that methylmercury behaves as an uncoupler. In the case of methylmercury, the mechanism corresponds to the entry of methylmercury as an undissociated, electroneutral $\mathrm{CH}_{3} \mathrm{HgOH}$ compound, which tends to accumulate inside, the $\mathrm{pH}$ being acid in the matrix. Once inside, the $\mathrm{CH}_{3} \mathrm{HgCl}^{+}$cation is extruded from the positiveinside potential, thus giving rise to a cyclic mechanism, resulting in the transport of a proton during any cycle, as in the case of uncouplers. The cyclic mechanism clearly explains, as it also does in the case of uncouplers, the low doses of methylmercury necessary to induce a fast internal $\mathrm{pH}$ alkalinization.

This mechanism, as already been mentioned, does not exclude the use of the antiporter mechanism (mechanism e) in the presence of chloride ions.

However, the possibility that $\mathrm{CH}_{3} \mathrm{HgCl}$ induces not only an uncoupling transport mechanism, but also a vacuolar ATPase inhibition, cannot be excluded. This is supported by the experiment shown in Fig. 1 (trace c), where the addition of $\mathrm{CH}_{3} \mathrm{HgCl}$ after that of bafilomycin induces the same alkalinization rate as that which occurs in the presence of bafilomycin alone (Fig. 1, trace a). A direct measurement of ATPase inhibition by $\mathrm{CH}_{3} \mathrm{HgCl}$ has been performed (Fig. 3) in order to clarify this point. The graph shows that $\mathrm{CH}_{3} \mathrm{HgCl}$ actually does inhibit the ATP hydrolysis, but the doses necessary to inhibit the ATPase are higher than those necessary to induce the alkalinization rate shown in Fig. 1 (trace a). This is demonstrated by the given data in Fig. 3, in which $20 \mu \mathrm{M} \mathrm{CH} \mathrm{CH}_{3} \mathrm{HgCl}$ induces only a

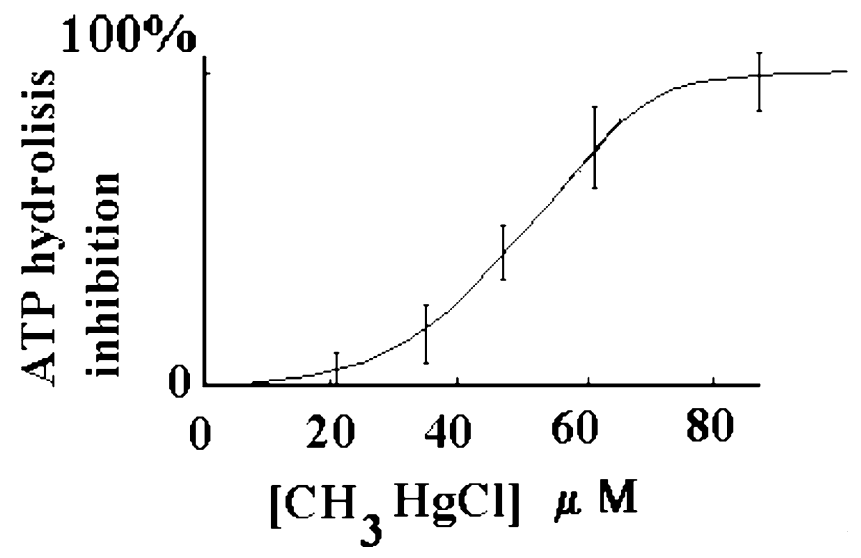

Fig. 3. The vacuolar ATPase inhibition induced by $\mathrm{CH}_{3} \mathrm{HgCl}$. The graphic reports the inhibition of the ATP hydrolysis induced by increasing amounts of $\mathrm{CH}_{3} \mathrm{HgCl}$. Experimental conditions: Medium composition: $0.125 \mathrm{M} \mathrm{KCl}, 0.5 \mathrm{mM}$ Hepes-Mops $\mathrm{pH}$ 7.4, $10 \mathrm{mM}$ $\mathrm{MgCl}_{2}$, oligomycin $2 \gamma, \mathrm{FCCP}, 60 \mathrm{nM}$. Each point in the graph (the mean value obtained after five replicate measurements) is the initial acidification rate obtained after addition of $400 \mu \mathrm{M}$ ATP to the medium $(4 \mathrm{ml})$ containing the lysosomes $\left(0.3 \mathrm{mg} \mathrm{ml}^{-1}\right)$ in the presence of various amounts of methylmercury. As reference point, the acidification rate was measured in the presence of $2 \mathrm{nM}$ bafilomycin. negligible effect on the ATPase. This also demonstrates that the toxic effect of $\mathrm{CH}_{3} \mathrm{HgCl}$ (with the lowest dose) is the uncoupling effect.

The importance of the possibility that $\mathrm{CH}_{3} \mathrm{HgCl}$ can behave as an uncoupler arises from the fact that an uncoupling mechanism necessarily implies the passage through the membrane of a charged compound, in this case $\mathrm{CH}_{3} \mathrm{Hg}^{+}$. This is made possible since under the operating conditions, the concentration of methylmercury in the supernatant of energized lysosomes (measured by means of atomic absorbtion after the centrifugation of the lysosomes) results as $8 \mu \mathrm{M}$. This implies that not all methylmercury added is bound to the membrane proteins and that the free methylmercury is available to be used in the uncoupling mechanism, as mentioned above.

The transport of the $\mathrm{CH}_{3} \mathrm{Hg}^{+}$cation can explain not only the toxicity (in mitochondria, where the $\mathrm{Ca}^{++}$ release and other correlated phenomena can be seen to be due to an uncoupling effect) but, in particular, the neurotoxicity, since the passage of the ion through a membrane occurs by means of a potential-driven mechanism. The above mentioned passage depends exponentially on the applied potential [37] and nerve cells possess the highest membrane potential in the cytoplasmic membrane. For this reason, we retain that only the uncoupling mechanism, even though it is probably not the only cause of toxicity, can explain why nerve cells are the preferential target for methylmercury.

\section{Supplementary material}

The material is available from the authors on request.

\section{References}

[1] T. Takeuchi, N. Norikawa, H. Matsumoto, Y. Shiraishi, Acta Neuropathol. 2 (1962) 40.

[2] F. Bakir, S.F. Damluji, L. Amin-Zaki, M. Murtadha, A. Khalidi, N.Y. Al-Rawi, S. Tikriti, H.I. Dahair, T.W. Clarkson, J.C. Smith, R.A. Doherty, Science 182 (1973) 230.

[3] C.R. Adams, D.K. Ziegler, J.T. Lin, J. Am. Med. Assoc. 250 (1983) 642.

[4] J.C. Hansen, in: A.W. Fumess, P.S. Rainbow (Eds.), Heavy Metals in the Marine Environment, CRC Press, Boca Raton, FL, 1990, pp. 227-243.

[5] L.W. Chang, in: P.S. Spencer, H.H. Schauenburg (Eds.), Experimental and Clinical Neurotoxicology, Williams and Williams Co, Baltimore, MD, 1980, pp. 508-526.

[6] L.W. Chang, Environ. Res. 14 (1977) 329.

[7] H. Komulainen, S.C. Bondy, Toxicol. Appl. Pharmacol. 88 (1987) 77.

[8] M.F. Hare, W.D. Atchinson, Toxicologist 12 (1992) 313.

[9] T. XiaoXia, T. Cuye, A. Castoldi, L. Manzo, L. Costa, J. Toxicol. Environ. Health 38 (1993) 159.

[10] A.H. Close, T.L. Guo, B.J. Shuker, Toxicol. Sci. 48 (1999) 68 
[11] B.J. Shenker, T.L. Cuo, J.M. Shapiro, Environ. Res. 77 (1998) 149.

[12] N. Sone, M. Larsstuvold, M. Kagawa, J. Biochem. 82 (1977) 859.

[13] M.A. Verity, W. Brown, M. Cheung, J. Neurochem. 25 (1975) 759.

[14] W. Atchinson, M. Hare, FASEB J. 8 (1994) 622.

[15] S.C. Bondy, M. McKee, Toxicol. Lett. 58 (1991) 13.

[16] M.F. Denny, M.F. Hare, W. Atchison, Toxicol. Appl. Pharmacol. 122 (1993) 222.

[17] B.J. Shenker, T.L. Gue, J.M. Shapiro, Toxicol. Appl. Pharmacol. 157 (1999) 23

[18] M. Bragadin, D. Marton, S. Manente, M. Grasso, A. Toninello, J. Inorg. Biochem. 89 (2002) 159.

[19] P.L. Savant, S. Shibo, U.S. Kumta, A.L. Tappel, Biochim. Biophys. Acta 85 (1964) 82.

[20] M.K. Pal, M. Schubert, J. Am. Chem. Soc. 84 (1962) 4384

[21] O.H. Lowry, N.J. Rosenbroug, A.L. Farr, R.J. Randall, J. Biol. Chem. 193 (1951) 265.

[22] N.I.T. Nishimura, B. Chance, Biochim. Biophys. Acta 84 (1962) 177.

[23] Y. Moriyama, M. Maeda, M. Futai, FEBS Lett. 302 (1992) 18.

[24] P. Dell'Antone, M. Bragadin, P. Zatta, Biochim. Biophys. Acta 1270 (1995) 137.
[25] P. Dell'Antone, R. Colonna, G.F. Azzone, Eur. J. Biochem. 24 (1972) 553.

[26] J. Mellman, R. Fuchs, A. Helenius, Annu. Rev. Biochem. 55 (1986) 663.

[27] P. Dell'Antone, Biochem. Biophys. Res. Commun. 86 (1979) 180.

[28] S. Ohkuma, Y. Moriyama, T. Takano, J. Biochem. 94 (1983) 1935.

[29] M. Bragadin, D. Marton, G. Scutari, P. Dell'Antone, J. Inorg. Biochem. 78 (2000) 205.

[30] P.O. Seglen, Methods Enzymol. 96 (1983) 737.

[31] E.J. Bowman, A. Siebers, K. Altendorf, Proc. Natl. Acad. Sci. USA 85 (1988) 7922.

[32] D.L. Stone, X.S. Xie, E. Racker, J. Biol. Chem. 258 (1984) 2701.

[33] W.N. Aldridge, B.W. Street, D. Skilleter, Biochem. J. 168 (1977) 353.

[34] D.N. Skilleter, Biochem. J. 146 (1975) 465.

[35] F.E. Brinckman, J.M. Bellama, in: F.E. Brinckman, J.M. Bellama (Eds.), Organometals and Organometalloids. Occurrence and Fate in the Environment, 17th ACS Symposium Series Meeting of the American Chemical Society, Anaheim, California, 1978.

[36] U. Bellucco, M. Giustiniani, M. Graziani, J. Am. Chem. Soc. 89 (1967) 6494.

[37] P. Lauger, Science 178 (1972) 24. 